\title{
VLBI OBSERVATIONS OF NGC4151 AT 6 CM WAVELENGTH
}

\author{
E. Preuss and W. Alef \\ Max-Planck-Institut für Radioastronomie \\ Auf dem Hügel 69 \\ D-5300 Bonn 1 \\ F.R.G.
}

\author{
A. Pedlar \\ Nuffield Radio Astronomy Laboratory \\ Jodrel1 Bank \\ Macclesfield, Chesire SK11 9DL \\ England
}

The fraction of Seyfert galaxies containing linear radio sources is quite substantial and may be more than 50\% (Ulvestad and Wilson 1984). About 20 of the well resolved Seyfert galaxies show double or triple structure on the arcsec scale. In this respect these objects are very reminiscent of the classical radio galaxies, but they are typically smaller than these by several orders of magnitude, both with regard to power $\sim 10^{39}-10^{41} \mathrm{erg} / \mathrm{s}$ and linear extent $\approx 1 \mathrm{kpc}$ (Wilson, this volume). They are basically confined in the wider nuclear environment. The central small scale ( $\lesssim$ 0!'1) radio sources in Seyfert galaxies are very weak, $\lesssim 10 \mathrm{mJy}$ at $6 \mathrm{~cm}$ wavelength, and have therefore hardly been observed with VLBI (see, e.g. Preuss 1984).

Important questions arising in view of the overall radio characteristics of Seyfert galaxies are: Do we see here a smaller and weaker version of the strong radio source phenomenon or are the physical processes at least partly qualitatively different? Is the energy supplied to the extended radio sources by continuous collimated outflow, by "jets", or by a more discontinuous process? Does accretion onto a compact massive object or enhanced star formation, provide the primary energy?

NGC4151, a Seyfert galaxy of type 1.5 (Osterbrock, this volume), is a particularly promising object to study these questions. It is the brightest Seyfert galaxy in several wavebands, and has been observed extensively from radio to $\gamma$-ray frequencies (e.g. Perola et al. 1982, Ulrich et a1. 1985). The distance is $20 \mathrm{Mpc} \cdot\left(50 / \mathrm{Ho}_{\mathrm{o}}\right)$ giving a scale ratio of 1 pc per 10 milliarcsec, so that a spatial resolution of $\sim 1 \mathrm{pc}$ can be achieved with the European VLBI Network at $\lambda \lesssim 6 \mathrm{~cm}$.

The total flux density of NGC4151 at $6 \mathrm{~cm}$ wavelength is $125 \mathrm{mJy}$. Observations made with the VLA at $20 \mathrm{~cm}$ (Johnston et al. 1982), at $6 \mathrm{~cm}$ (Ulvestad et al. 1981), and at $2 \mathrm{~cm}$ (Wilson and Ulvestad 1982) and 
observations made with MERLIN at $18 \mathrm{~cm}$ (Booler et al. 1982) have shown that the radio source has a linear structure $\sim 3 . .5$ in extent at p.a. $\sim 77^{\circ}$, with a central double source of separation 0.45 at p.a. $\sim 83^{\circ}$. VLBI observations made at $18 \mathrm{~cm}$ by Harrison et al. (1986) resolved this double source into a collinear triple structure of the same overall extent.

Pilot observations made with the narrow band (2 MHz) VLBI system (Preuss and Fosbury 1983) showed that about $20 \mathrm{mJy}$ originate from the 10 milliarcsec scale at $6 \mathrm{~cm}$ wavelength. We report here the first VLBI observations with the broad band ( $56 \mathrm{MHz}$ ) system made in 1985.4 in the band 4954 to $5010 \mathrm{MHz}$. This 5-station experiment was troubled by a number of technical failures so that we obtained useful data only from the three baselines connecting Medicina (Italy), Effelsberg (W.-Germany), and Westerbork (The Netherlands). The angular resolution $(0.5 \lambda / \mathrm{D})$ thus obtained was $\sim 0$ "'006 or $0.6 \mathrm{pc}$. The rms noise on the most sensitive baseline (Effelsberg - Westerbork) was $\sim 1 \mathrm{mJy}$. By means of a new fringe fitting procedure which utilizes antenna based residuals of delay and fringe rate (Alef and Porcas 1986) we were able to push the detection limit for the interference fringes down to the noise level. The image restauration was done in two steps. First a two component model was fitted to the measured data. This was then used as initial model for a self calibration mapping procedure (developed by J.D. Romney), in which the iteration process was confined to narrow windows around the two components of the initial model.

The resulting map is shown in Fig. 1. It is very similar to the

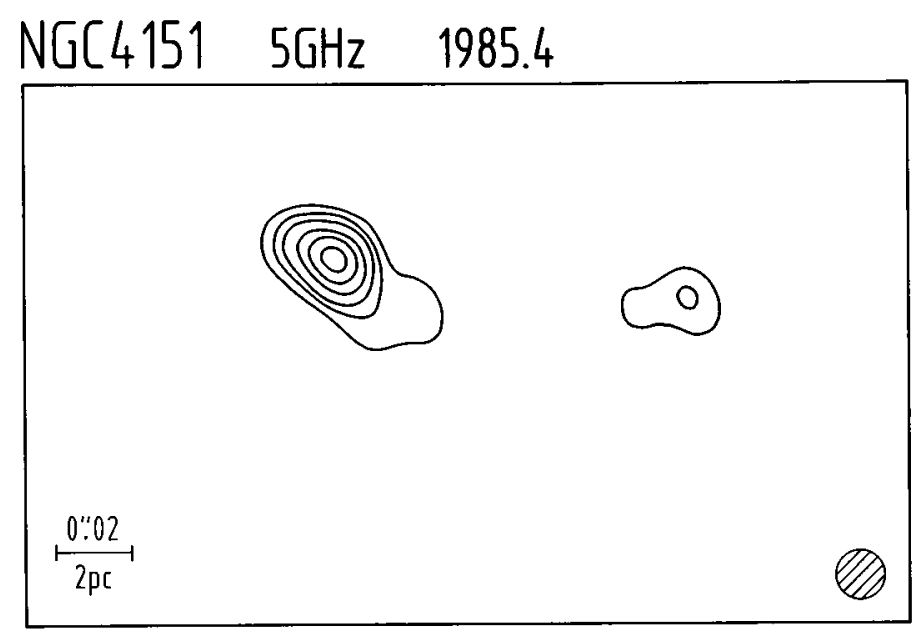

Fig. 1: VLBI map of the core of NGC4151 at $6 \mathrm{~cm}$ for epoch 1985.4. The smoothing beam is shown as a shaded circle. Contour levels are $10,20,30,50,70,90$ per cent of the peak brightness ( $\left.3.4 \times 10^{7} \mathrm{~K}\right)$. 
Eastern part of the $18 \mathrm{~cm}$ VLBI map by Harrison et al. (1986). The total flux in this map is $15 \mathrm{mJy}$. The separation of the double source is 0.'092 at p.a. $83^{\circ}$, in agreement with the $18 \mathrm{~cm}$ map. The brighter Eastern component is elongated along p.a. 570, and with some caution one notices a bend towards the Western component. Comparison of the $6 \mathrm{~cm}$ and $18 \mathrm{~cm}$ VLBI maps with the aim to obtain spectral index information can only be done with extreme care, because of the strong resolution effects at $6 \mathrm{~cm}$. Although we cannot give accurate numbers for the spectral indices, we can state, however, that the spectrum of the Eastern component as a whole must be flatter than that of the Western component. This suggests that the pc scale radio structure is one-sided, with the Eastern component possibly being the "core". The linear structure of both VLBI maps and the fact that all discernible components are elongated themselves in the direction of the overall source axis (allowing for a bend on the pc scale), strongly supports the hypothesis (Booler et al. 1982, Wilson and Ulvested 1982) that collimated ejection or outflow is occurring. Our map shows that a first collimation must occur on a scale $\lesssim 1$ pc.

This observation demonstrates that with a sensitive VLBI array, the pc-scale structure of NGC4151 can be mapped at $6 \mathrm{~cm}$ with a dynamic range higher than achieved here. This opens the possibility of monitoring the small scale radio structure and measuring any relative proper motion $\gtrsim 30000 \mathrm{~km} / \mathrm{s}$.

\section{REFERENCES}

Alef, W., Porcas, R.W.: 1986, Astron. Astrophys. in press

Booler, R.V., Pedlar, A., Davies, R.D.: 1982, Monthly Notices Roy. Astron. Soc. 1999,229

Harrison, B., Pediar, A., Unger, S.W., Burgess, P., Graham, D.A., Preuss, E.: 1986, Monthly Notices Roy. Astron. Soc. 21 18,775

Johnston, K.J., Elvis, M., Kjer, D., Shen, B.S.P. . 1982, Astrophys. J. $\underline{\underline{26}} \underline{\underline{\underline{2}}, 61}$

Perola et a1.: 1982, Month1y Notices Roy. Astron. Soc. 200, 293

Preuss, E.: 1984, Proc. IAU Symp. No. 110, 251

Preuss, E., Fosbury, R.A.E.: 1983, Monthly Notices Roy. Astron. Soc. $2 \underline{\underline{0}} \underline{=}, 783$

Ulrich, M.H. et a1.: 1985, Nature $\underline{\underline{3}} \underline{\underline{1}} \underline{\underline{3}}, 747$

Ulvestad, J.S., Wilson, A.S., Sramek, R.A.: 1981, Astrophys. J. 247, 419

Ulvestad, J.S., Wilson, A.S.: 1984, Astrophys. J. $2 \frac{8}{5}, 439$

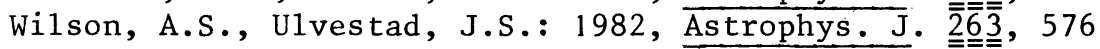



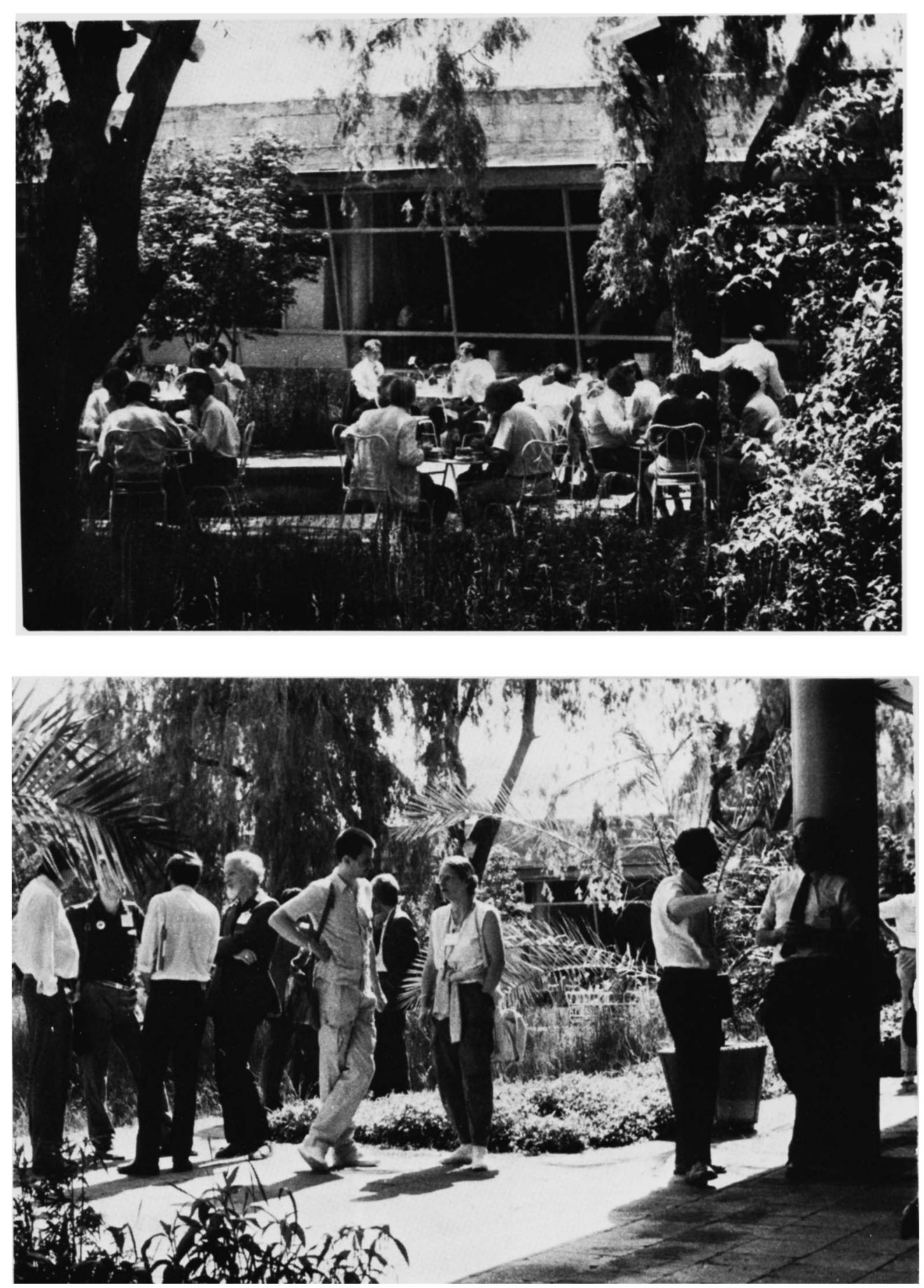

Some examples of Very Lively Byurakan Interactions (VLBI) 